\title{
Highlights of the 3rd International Conference on High Pressure Bioscience and Biotechnology
}

J.A. Mignaco ${ }^{1}$, L.M.T.R. Lima²,

A. Rosenthal ${ }^{3}$,

D. Foguel ${ }^{1}$ and J.L. Silva ${ }^{1}$
${ }^{1}$ Instituto de Bioquímica Médica, ${ }^{2}$ Faculdade de Farmácia, Centro de Ciências da Saúde, Universidade Federal do Rio de Janeiro, Rio de Janeiro, RJ, Brasil

${ }^{3}$ Embrapa Agroindústria de Alimentos, EMBRAPA, Rio de Janeiro, RJ, Brasil

\section{Correspondence}

J.L. Silva

Instituto de Bioquímica Médica Centro Nacional de Ressonância Magnética, UFRJ

21941-590 Rio de Janeiro, RJ

Brasil

E-mail: jerson@bioqmed.ufrj.br

Research supported by CNPq, FINEP, FAPERJ, CAPES, FUJB, FECD, EMBRAPA and UFRJ.

Received June 17, 2005 Accepted July 11, 2005

\section{Abstract}

The 3rd International Conference on High Pressure Bioscience and Biotechnology was held in the city of Rio de Janeiro from September 27 to September 30, 2004. The meeting, promoted by the International Association of High Pressure Bioscience and Biotechnology (IAHPBB), congregated top scientists and researchers from all over the world. In common, they shared the use of hydrostatic pressure for research, technical development, or industrial applications. The meeting consisted of invited lectures, contributed papers and a well-attended poster session. Very exciting discussions were held inside and outside the sessions, and the goals of discussing state-of-the-art data and establishing working collaborations and co-operations were fully attained.

\section{The Conference on High Pressure Bioscience and Biotechnology (HPBB2004)}

HPBB2004 was the third conference promoted by the International Association of High Pressure Bioscience and Biotechnology, established in 1998. The first official conference was held in Kyoto, Japan, in 2000, the second in Dortmund, Germany, in 2002, and the 4th is due to be held
Key words

- Hydrostatic pressure

- Phase diagram

- Protein folding

- Food technology

- Piezotolerance
- Compressibility 
ogy, and engineering, who had the opportunity to present their research, as well as to exchange ideas and comment upon the latest developments in High Pressure Bioscience and Biotechnology. Topics such as high pressure (HP) studies on proteins, nucleic acids and polyelectrolytes, lipids and biomembranes, HP enzymology, HP biophysics and solvent effects, HP effects on living matter, HP and marine biology, HP food science and technology, HP general applications in bioscience, HP techniques, HP engineering, and HP in medical science were extensively discussed.

Since historical times life and biological phenomena on earth have been studied from an anthropocentric perspective, and mankind misjudged - or neglected - the fact that most of the earth's surface is indeed situated underwater. Most of the biosphere is submitted to pressures above that considered to be the "normal atmospheric pressure" of $0.1 \mathrm{MPa}$ (1). For a long time the fact that pressure was an important physicochemical parameter was empirically known, and in the 17 th century Blaise Pascal established the basis for the explanation of pressure effects on fluids and gases. However, until the 20th century the effects of pressure on living matter were underestimated or unknown due to severe technical limitations imposed by the lack of materials and technology that could sustain pressures ranging from a few to $100 \mathrm{MPa}$. In $1895, \mathrm{H}$. Royer (2) reported the killing of bacteria by hydrostatic pressure, while in 1899 , B.H. Hite and coworkers (3) reported that pressure treatment could improve milk preservation. That pressure would interact with biological mol- ecules to produce effects similar to those of other physicochemical parameters was demonstrated by the seminal work of P.W. Bridgman in 1914 (4), who observed that high hydrostatic pressure produced coagulation of egg white resulting in an end product similar to that achieved by heating or by acid treatment. Because any increase of pressure on a fluid in equilibrium is transmitted integrally to all the points of the fluid, pressure can be rapidly and homogeneously applied to or removed from a liquid sample in a time- and energy-saving manner. Therefore, the inconvenient formation of temperature or chemical gradients is avoided, preserving homogeneity of the samples treated with pressure.

Today, generators and devices able to withstand hydrostatic pressures of thousands of MPa find a panoply of applications, greatly exceeding those imagined by common persons. Hydrostatic pressure is a reality for food science and technology, both for food preparation and preservation. In 1989, food companies introduced pressure for the production/preservation of jellies, marmalades, meat, ham, fruit juices, avocado paste (guacamole), and oysters, to mention a few. The understanding of the effects of pressure on biomolecules is expanding the use of hydrostatic pressure for the medical, pharmaceutical, industrial, biotechnological, and even cosmological fields.

In this brief overview of the HPBB2004, we will only comment on the subjects presented by the invited lecturers, as a way to highlight some of the current relevant topics in High Hydrostatic Pressure Bioscience and Biotechnology. However, very excit- 
ing presentations and discussions were a general rule for the oral contributions (a total of 26) and for the poster session (60 posters). The abstracts of these presentations are available in PDF format at the HPBB2004 homepage (http://www. bioqmed.ufrj.br/hpbb2004).

The opening session of the HPBB2004 was presided by Dr. Jerson L. Silva, Chairman of the Conference. Many local authorities and representatives of the government, financial sponsors and scientific entities that supported the conference were present. In this session the relevance of High Pressure in Biosciences as a state-ofthe-art methodology in the life sciences was strongly emphasized. The session was energized by the Opening Lecture, presented by Dr. Karel Heremans, from the Katholieke Universiteit Leuven (Belgium), who talked about Protein Dynamics and the roles of hydration and cavities for protein structure (5). Dr. Heremans, who received a special Honor from the HPBB2004 organizers during the meeting in recognition of his contributions to the field of High Pressure Research in Biosciences, discussed the thermodynamic aspects of the temperature-pressure behavior of proteins in solution, and the relevance of protein compressibility, volume changes, hydration, and cavities for the understanding of the folding and unfolding processes in proteins (5). According to Le Chatelier's principle, any increase in pressure on a system (an aqueous protein solution, for example) will force this system to the smallest possible volume, thus initially leading to limited compression of the protein's cavities and thereafter forcing water into these cavi- ties (for a review, see Ref. 6). This leads either to dissociation of protein subunits or to partial or total unfolding of proteins. The pressure needed to dissociate and/or unfold each different protein varies, and depends less on the size than on the strength of interactions inside the protein or between the subunits that form it. The novel results obtained by Dr. Heremans' group for the pressure-unfolding pathway of lysozyme with the novel technique of positron annihilation lifetime spectroscopy were presented and discussed. The phenomenon of aggregation of proteins to form fibrils and amyloid aggregates was discussed in the light of results obtained from pressure studies that demonstrated that highly soluble proteins could be converted to insoluble fibers rich in B-pleated structures.

As pointed out in several presentations during the conference and reported in this special issue, conformational diseases and disorders caused by protein misfolding and/ or aggregation are among the subjects of most intense research in the high pressure field. This is so not only because comprehension of the routes and factors that lead to protein aggregation could help develop clinical and pharmaceutical approaches to combat these disorders (which include Alzheimer's disease, prion diseases, Parkinson's disease, and systemic amyloidosis, to mention a few), but also because with the aging of the world's population these disorders present an alarmingly increasing incidence.

Prof. Kazuyuki Akasaka (Kinki University, Japan) discussed the Probing of Protein Structural Changes by the Use of 
Variable Pressure Nuclear Magnetic Resonance. He showed how this technique could probe semi-stable conformers, which are populated by high pressure and which are involved in protein function and folding. The data were discussed in light of the funnel-type energy landscape pictured for the conformational states of a protein, which, from a broad spectrum of possible conformations, tend to be reduced to a progressively restricted number of energetically favored (allowed) conformations. The presentation by Dr. Lazlo Smeller (Semmelweis University, Hungary) emphasized the relevance of pressure-unfolded proteins as useful objects for folding-misfolding and aggregation studies. Results from his group showed that metastable intermediates of myoglobin and horseradish peroxidase, with a high tendency to aggregate, appeared upon partial refolding from the pressure-denatured state. Prof. Débora Foguel (Universidade Federal do Rio de Janeiro, Brazil) talked about the amyloidogenic protein transthyretin, which is involved in senile systemic amyloidosis and familial amyloidotic polyneuropathy. The high hydrostatic pressure aggregation protocol is used by this group to screen different anti-amyloidogenic compounds, which include well-known, nonsteroid anti-inflammatory drugs. Similar experiments with Parkinson's $\alpha$-synuclein were mentioned in the discussion of whether small aggregates rather than the mature amyloidogenic fibrils are the relevant species involved in pathogenesis.

Prof. Claude Balny (INSERM-Montpellier, France) discussed the Powerful High Pressure Tool for Protein Conforma- tional Studies (7). Dr. Balny presented a comprehensive discussion of data from his own and other groups, covering the effects of high hydrostatic pressure on the secondary, tertiary and quaternary structures of proteins, on enzyme kinetics for monomeric and oligomeric cases, and on the phenomenon of protein aggregation, amyloidosis and prion diseases (7).

Dr. Brian G. Lefebvre (Rowan University, USA) talked about Engineering Approaches for Combating Protein Aggregation. Formation of protein aggregates is a problem not only for human and animal health, but also for the biotechnology industry since high throughput production of relevant proteins is sometimes hampered by formation of protein aggregates and inclusion bodies.

The Role of the Waters of Hydration in Protein Structure, Stability and Dynamics was highlighted by Dr. Catherine A. Royer (CBS-Montpellier, France) (8). Perturbation by high pressure directly involves changes in the volume of a system and therefore can give information about the role of water, and particularly of those water molecules involved in the hydration of the protein that contribute to protein structure, stability and dynamics (8). Dr. Sérgio T. Ferreira (UFRJ, Brazil) (9) talked about the detection of metastable, partially folded intermediates in the folding and misfolding of proteins. Dr. Ferreira's group uses a combination of hydrostatic pressure, low/high temperatures and chemical denaturants to enable the detection and characterization of partially (un)folded intermediates, and has reported results on the pathways and energetics for the de 
novo folding of designed three- and fourhelix bundle, prion protein, and human lysozyme variants (9).

In the theoretical field, Dr. Angel Garcia (Los Alamos National Laboratory, USA) described the determination of folding/unfolding of the C-terminal (41-56) fragment of $G$ protein as a function of density and temperature using replica exchange molecular dynamics simulations of an atomic model of the solvated oligopeptide, considering the effects of temperature and pressure. This study was recently published in the Proceedings of the National Academy of Sciences, USA (10). For pressure denaturation, the weakening of the hydrophobic interaction between the bulky amino acid side chains was found to be crucial at lower temperatures, leading to an apparent destabilization of the folded backbone structure at elevated pressures (10). These theoretical calculations are consistent with calculations derived from experimental findings described by several groups for cold-assisted pressure denaturation (5-7).

Prof. Shigeru Kunugi (Kyoto Institute of Technology, Japan) stressed his groups' observations of differences in the pressure and temperature transitions for proteins and polymeric gels (11). Pressure- and temperature-driven protein transitions have many similarities and are normally summarized in simple thermodynamic equations. Using different polymer gels (namely poly(N-vinylisobutyramide) cross-linked with butylene-bis-N-vinylacetamide), Prof. Kunugi's group found that in temperature transitions the gels showed drastic volume shrinkage and decreasing transparency, while for pressure transitions transparency was recovered in spite of the shrinking volume. According to Prof. Kunugi, similar observations have been reported for proteins. Since proteins ultimately are polymers, the authors called attention to the need to reinvestigate and better characterize the differences and similarities of these two thermodynamic transitions at the molecular level (11).

Dr. Suzanne F. Scarlata (State University of New York, Stony Brook, USA) reported on The Effects of Pressure on the Activated State of Phospholipase C- $\beta$ and its Interaction with G Protein Subunits (12). Membrane-bound, G-protein-regulated phospholipase C- $\beta$ (PLC- $\beta$ ) metabolizes PIP-4,5-bisphosphate and modulates intracellular calcium concentrations and protein kinase $\mathrm{C}$ activities, and its activity is increased by pressure, as observed with previously studied PLC- $\Delta$. Dr. Scarlata's group showed that, as is the case for other interfacial enzymes, membrane binding was pressure-independent, that high hydrostatic pressure activated PLC- $\beta$ by hydration/conformation changes at the catalytic core, and moreover that, in the presence of the membrane, association with the regulatory G-protein was favored and that pressure up to $2 \mathrm{kbar}$ did not increase hydration of the membrane interface (12). This was interpreted to indicate that the presence of membranes stabilized the protein complex due to the protein confinement on its surface, therefore pointing to a role of a reduced volume in the lateral association between membrane proteins.

This presentation was followed by that of Prof. Roland Winter (University of Dortmund, Germany), Exploring the Con- 
figurational Landscape of Biomolecules Under High Pressure Conditions: From Lipid Membranes to Proteins. Dr. Winter presented a wide array of data on the influence of high hydrostatic pressure on the polymorphic phases of lipid bilayers at different $\mathrm{pH}$ and temperatures, obtained by several spectroscopic and analytical techniques, and emphasized the relevance of this knowledge for understanding life at high pressure and also for developing biotechnological applications (13). The flip side of protein folding and unfolding by high hydrostatic pressure and temperature was also explored from a physicochemical point of view using membrane proteins and soluble proteins as models, and a threedimensional free energy surface diagram for protein stability was presented (13). Prof. Jerson L. Silva presented New Insights into the Mechanism of Protein Folding and Misfolding Derived from Pressure Studies, with an emphasis on the prion diseases (14). Protein misfolding can lead to conformational diseases such as amyloidosis, Alzheimer's, Parkinson's, prion and tumor diseases in humans, related diseases in animals (such as ovine scrapie or bovine spongiform encephalitis), or formation of inclusion bodies of proteins overexpressed in bacteria. Thus, high hydrostatic pressure obviously is a powerful technique regarding the attempts to develop therapies for the disease-related misfolding of proteins and strategies to disperse inclusion bodies of proteins of biotechnological relevance. Furthermore, the use of high hydrostatic pressure promises to contribute to the identification of the mechanisms underlying these defects and to the development of therapies against these diseases (14). Dr. Igor Polikarpov (Institute of Physics, São Carlos, USP, Brazil) presented a Study on the Multimeric Forms of Retinoic Acid x Receptor in Solution. Human retinoid-X receptor ( $\mathrm{XXR}$ ) proteins in solution may exist as monomers, dimers, and tetramers. Dr. Polikarpov's group studied the structural organization of human RXR by solution synchrotron X-ray scattering, and showed dissociation of RXR oligomers by high hydrostatic pressure monitored by fluorescence spectroscopy.

The Effects of Pressure on Polymers and Foods were then discussed. Prof. Horst Ludwig (Universität Heidelberg, Germany) showed in his talk that polymer films used for food storage present modified barrier properties under high pressure (15). Using food-encountered molecules of different polarities as model substances, his group demonstrated that in most cases the polymer films' permeability is reversibly decreased at high pressure, with no sign of mechanical damage except for a tendency to delaminate. If polymers were to be optimized, this might prove of great relevance for the future treatment of pre-packed foods with high hydrostatic pressure. Next, Dr. Amauri Rosenthal (CTAA-EMBRAPA Director, Brazil) presented the actions taken by EMBRAPA to develop pressure-treated juices and introduce them on the Brazilian market. The encouraging results with pineapple juice and other tropical fruit products and the good acceptance by the public were a high point for the high hydrostatic pressure food-processing technology, demonstrating its commercial viability due to a still unexploited niche of products. The 
thermo- and fluid-dynamics of high pressure-induced phase transitions in lipid substances was the subject of Dr. Christoph Hartmann's presentation. He described a new approach that may prove to be of great relevance for the food industry, where the treatment of different water-oil mixtures is increasingly used in pressure-assisted freezing and thawing for food preparation. Treating a diverse polymer subject, Dr. Tigran Chalikian (University of Toronto, Canada) discussed the interactions of nucleic acids with different monovalent cations, and how these reciprocally interact with the hydration shell of the cations and polymers.

With the growing interest in deep-sea biology and pressure effects on living matter, there has been an increasing effort to better understand not only how pressure affects proteins and lipids, but also how pressure regulates metabolism and gene expression in living organisms. Prof. Douglas H. Bartlett (University of California, San Diego, USA) talked about the functional genomics of high-pressure adaptation in the deep sea bacterium Photobacterium profundum. The complete genome and comparative and functional genomic experiments, together with transcriptome studies, were described, leading to a definition of the genes involved in adaptation to high pressure. Part of this work was recently published in Science (16). From these results, it appears that high pressure adaptation requires modulation of membrane and cell wall biosynthesis, amino acid utilization, $\mathrm{pH}$ homeostasis, presence of multiple cytochrome and ATP-synthase systems, as well as particular sets of isozymes. In contrast, cold adaptation requires changes in membrane structure, nucleic acid processing, protein secretion, glycolysis and TCA cycle enzyme expression, as well as protein turnover and translation. Prof. Rudi F. Vogel (Technische Universität München, Germany) introduced the subject of high pressure-sensitive gene expression in Lactobacillus sanfranciscensis, a lactic acid bacterium used in food biotechnology (17). The pressure stress response was analyzed by changes in the proteome (by two-dimensional-electrophoresis), transcriptome with microarrays and real time PCR. More than 25 proteins were identified that are differentially expressed upon high pressure stress, but no specific pressure stress response protein was identified when compared to different types of stress. From these results, it was hypothesized that the cells try to compensate for the impaired membrane transport and translation by an up-regulation of some specific genes (16). Akin data were reported by Dr. Patricia Fernandes (UFES, Brazil) concerning Pressure Response in the Yeast Saccharomyces cerevisiae (18). While a pressure of $50 \mathrm{MPa}$ did not induce visible cell damage or loss of viability, high hydrostatic pressure $>100 \mathrm{MPa}$ decreased cell viability and inactivated yeast cells at $220 \mathrm{MPa}$. In addition to damage to the cell wall, cytoskeleton, cell membranes and organelles, the pressure response involved the up-regulation of several genes involved in stress defense and carbohydrate metabolism, and down-regulation of genes related to cell cycle progression and protein synthesis.

Combining microbiology and the food field, inactivation of microorganisms in 
food preparation is an advantage of high pressure treatment. Prof. Bernhard Tauscher (Federal Research Center on Nutrition \& Food, Karlsruhe, Germany) discussed results showing that food of high sensory quality and statistical sterility was produced by the combination of pressure and temperature, while most of the vitamins, peptides, food additives, metal ions, and compounds relevant for the sensory characteristics of food products were preserved. As an additional result, it was suggested that the effects of pressure on the prion protein might be an interesting methodology to prevent contamination by foodborne prion protein, since high hydrostatic pressure induces structural changes in the prion protein that make it susceptible to proteases (19).

The meeting was honored by and wishes to acknowledge the presence of an exposition booth from the manufacturer of scientific equipment TF Instruments - $\mathrm{GmbH}$, whose innovative technique of Ultrasonic Resonator Technology should prove of great value for the study of rheology, compressibility and phase transitions of solutes and solutions under high pressure. Hopefully the use of ultrasound for pres- sure studies will find a great deal of application not only for basic research, but also for food and nutraceuticals prepared with pressure in the near future. We also hope that worldwide Scientific Equipment suppliers and manufacturers will find interest in presenting their products at future Conferences on High Pressure Bioscience and Biotechnology in order to increase the interface with R\&D groups at private companies and universities in the growing field of High Pressure Research.

Finally, we would like to point out that the HPBB2004 was a very high-quality scientific meeting, where topics regarding the state-of-the-art for High Pressure Bioscience and Biotechnology, many of them in a close frontier with industrial, pharmaceutical and medical applications, were discussed in a very fruitful manner, and where young scientists were able to establish working collaborations with senior researchers and group leaders. We really expect the next meeting (HPBB2006) to be a great and expanding success. The organizers of the HPBB2004 wish to thank all people who contributed to making the 3rd International Conference on High Pressure Bioscience and Biotechnology a success.

\section{References}

1. Abe F \& Horikoshi K (2001). The biotechnological potential of piezophiles. Trends in Biotechnology, 19: 102-108.

2. Royer H (1895). Archives de Physiologie Normale et Pathologique, 7: 12.

3. Hite BH (1899). The effect of pressure in the preservation of milk. West Virginia Agricultural Experiment Station Bulletins, 58: 15-35.

4. Bridgman PW (1914). The coagulation of albumen by pressure. Journal of Biological Chemistry, 19: 11-12.

5. Heremans K (2005). Protein dynamics: hydration and cavities. Brazilian Journal of Medical and Biological Research, 38: 11571165.

6. Silva JL, Foguel D \& Royer C (2001). Pressure provides new insights into protein folding, dynamics and structure. Trends in Biochemical Sciences, 26: 612-618.

7. Marchal S, Torrent J, Masson P et al. (2005). The powerful high pressure tool for protein conformational studies. Brazilian Journal of Medical and Biological Research, 38: 1175-1183.

8. Royer CA (2005). Insights into the role of hydration in protein structure and stability obtained through hydrostatic pressure studies. Brazilian Journal of Medical and Biological Research, 38: 11671173.

9. Ferreira ST, Chapeaurouge A \& De Felice FG (2005). Stabilization of partially folded states in protein folding/misfolding transitions using hydrostatic pressure. Brazilian Journal of Medical and Biological 
Research, 38: 1215-1222.

10. Paschek D, Gnanakaran S \& Garcia AE (2005) Simulations of the pressure and temperature unfolding of an alpha-helical peptide. Proceedings of the National Academy of Sciences, USA, 102: 67656770 .

11. Kunugi S, Kameyama K, Tada T et al. (2005). Differences in pressure and temperature transitions of proteins and polymer gels. Brazilian Journal of Medical and Biological Research, 38: 1233-1238.

12. Scarlata $S$ (2005). The effect of hydrostatic pressure on membranebound proteins. Brazilian Journal of Medical and Biological Research, 38: 1203-1208.

13. Vogtt K \& Winter R (2005). Pressure-assisted cold denaturation of hen egg white lysozyme: the influence of co-solvents probed by hydrogen exchange nuclear magnetic resonance. Brazilian Journal of Medical and Biological Research, 38: 1185-1193.

14. Cordeiro Y, Kraineva J, Winter R et al. (2005). Volume and energy folding landscape of prion protein revealed by pressure. Brazilian Journal of Medical and Biological Research, 38: 1195-1201.
15. Schmerder A, Richter T, Langowski H-C et al. (2005). Effect of high hydrostatic pressure on the barrier properties of polyamide-6 films. Brazilian Journal of Medical and Biological Research, 38: 12791283.

16. Vezzi A, Campanaro S, D'Angelo $M$ et al. (2005). Life at depth: Photobacterium profundum genome sequence and expression analysis. Science, 307: 1459-1461.

17. Vogel RF, Pavlovic M, Hörmann S et al. (2005). High pressuresensitive gene expression in Lactobacillus sanfranciscensis. Brazilian Journal of Medical and Biological Research, 38: 1247-1252.

18. Fernandes PMB (2005). How does yeast respond to pressure? Brazilian Journal of Medical and Biological Research, 38: 12391245.

19. Heindl P, Fernández García A, Büttner M et al. (2005). Some physico-chemical parameters that influence proteinase $\mathrm{K}$ resistance and the infectivity of $\mathrm{PrPSc}$ after high pressure treatment. Brazilian Journal of Medical and Biological Research, 38: 1223-1231. 